\title{
La bactofugation du lait et l'élimination des spores de Clostridium tyrobutyricum
}

\author{
par \\ J. L. BERGERE \\ Station Centrale de Recherches Laitières et de Technologie des Produits \\ Animaux, C.N.R.Z, I.N.R.A. (78) Jouy-en-Josas
}

(avec la collaboration technique de D. LE BARS

et Mme J. COMMISSAIRE)

Parmi les Clostridium susceptibles d'être apportés par le lait, celui qui présente le plus grand danger pour la fromagerie est Clostridium tyrobutyricum, espèce appartenant aux Clostridium du groupe butyrique (Bergère, Gouet, Hermier et Mocquot, 1968). Sa croissance dans certains types de fromages (Gruyère, Edam, Gouda, etc.) provoque en effet un défaut connu depuis longtemps sous le nom de gonflement butyrique. L'apparition du gonflement butyrique dans les fromages est en relation étroite avec le nombre de spores de $\mathrm{Cl}$. tyrobutyricum initialement présentes dans le lait (Thomé et Swartling, 1953 ; Bergère et al., 1968 et 1969). Pour le fromage de Gruyère, le seuil critique se situe aux environs de 200 spores par litre. Habituellement inférieure à ce seuil la contamination du lait le dépasse dès que les animaux reçoivent dans leur alimentation certains fourrages ensilés ; elle peut même atteindre jusqu'à 100000 spores par litre lorsque la qualité de ces ensilages est très mauvaise (Bergère et al., 1968 et 1969).

L'apparition du défaut butyrique peut, bien entendu, être évitée en supprimant tout ensilage de la ration des vaches laitières et c'est ce qu'on a fait pendant longtemps. Mais on peut également limiter la contamination du lait en utilisant seulement certains type d'ensilage de bonne qualité bactériologique, tels que l'A.I.V., à l'instar des finlandais, et en prenant certaines précautions à la traite (Bergère et al., 1969). On peut enfin chercher à éliminer les spores des laits d'ensilage ; pour cela on a proposé d'utiliser la bactofugation. Ce procédé consiste essentiellement en l'élimination des bactéries du lait par la force centrifuge (environ $10000 \mathrm{~g}$ ) à une température appropriée qui est généralement une température de pasteurisation (Simonart et al., 1953, 1959). Les résultats obtenus varient suivant les bactéries et la température choisie pour la bactofugation. Pour 
les spores de Clostridium le pourcentage d'élimination est de 99,1 p 100 . à $78^{\circ} \mathrm{C}$ (Simonart et al., 1966), pour les spores de Clostridium du groupe butyrique (sans indication d'espèce) il varie mais dépasse généralement 90 p. 100 à $59-60^{\circ} \mathrm{C}$ (Lodin et al., 1965 et 1967 ; Syrjänen, 1963, 1964 a et b ; Peltola et Syrjänen, 1965). Ces résultats paraissent étonnants puisqu'on sait par ailleurs que dans du lait soumis à une simple centrifugation, les spores de "butyriques " se rassemblent plutôt dans la crème que dans le sédiment (Hostettler et al., 1941 ; Sjöström et al., 1963), vraisemblablement grâce à des agglutinines présentes dans le lait. Il est connu en effet que certaines protéines du lait sont des anticorps susceptibles d'agglutiner les bactéries et de jouer un rôle important en technologie laitière (Auclair, 1964). En particulier dans le lait entier laissé au repos, la crème qui s'accumule à la surface contient la plus grande partie des bactéries agglutinées ou fixées aux globules gras par les agglutinines acisorbées sur ces globules. Par contre, dans le lait écrémé, les bactéries agglutinées se déposent au fond du récipient.

Dans le présent travail nous avons cherché à préciser et compléter les données antérieures sur la bactofugation en utilisant des laits ensemencés avec des spores dans un état aussi bien déterminé que possible et provenant des souches connues de l'espèce Clostridium tyrobutyricum. Nous avons ainsi déterminé le taux d'élimination des spores en fonction de la température et sa reproductibilité en fonction des essais. Ensuite nous montrons que le lait contient effectitivement des agglutinines responsables de l'entraînement de ces spores avec les globules gras et susceptibles de jouer un rôle défavorable dans la bactofugation.

\section{MATERIEL ET METHODES}

\section{1) Bactofugation du lait}

Le bactofuge Alfa-Laval installé à la Laiterie Expérimentale du C.N.R.Z. était du type D. 3187 M. Les essais ont été effectués pendant la période hivernale (octobre à mai). Le lait utilisé, 3000 l par essai, était du lait de mélange conservé à $4^{\circ} \mathrm{C}$ pendant une nuit. Le matin ce lait était d'abord réchauffé à $40-45^{\circ} \mathrm{C}$ et épuré par centrifugation puis chauffé dans un pasteurisateur (temps de passage $30 \mathrm{~s}$ ) à une température légèrement supérieure $\left(0,5\right.$ à $\left.1^{\circ} \mathrm{C}\right)$ à celle prévue pour la bactofugation et enfin envoyé dans le bactofuge. A la sortie de cet appareil, le lait était refroidi et renvoyé dans une citerne. Le débit et la température de la bactofugation sont précisés dans les résultats.

\section{2) Ensemencement du lait en spores}

Avant réchauffage, le lait était toujours ensemencé avec une suspension de spores de $\mathrm{Cl}$. tyrobutyricum dans la citerne servant au stockage et brassé tout en étant pré-chauffé à $25-30^{\circ} \mathrm{C}$. Dans la plupart des cas la souche CNRZ $510 \mathrm{de} \mathrm{Cl}$. tyrobutyricum a été 
utilisée ; cependant quelques essais comparatifs ont été effectués avec les souches CNRZ 500, 509, 510 et 518 appartenant à la même espèce. Les nombres de spores sont indiqués dans les résultats. Ces spores sont préparées par la méthode de culture en boyau de dialyse qui a déjà été utilisée pour cette espèce (Cerf, Bergère et Hermier, 1967). Le milieu de culture est le milieu T.G.E. composé de : trypticase B.B.L., $10 \mathrm{~g}$; extrait de levure Difco, $1 \mathrm{~g}$; glucose, $10 \mathrm{~g}$; MgSO, $7 \mathrm{H}_{2} \mathrm{O}, 200 \mathrm{mg}$; $\mathrm{MnSO}_{4}, \mathrm{H}_{2} \mathrm{O}, 7 \mathrm{mg}$; $\mathrm{FeSO}_{4}, 7 \mathrm{H} \mathrm{H}_{2} \mathrm{O}, 10 \mathrm{mg}$; $\mathrm{CaCl}_{2}$, $12 \mathrm{H}_{2} \mathrm{O}, 73,5 \mathrm{mg}$; acide ascorbique, $1 \mathrm{~g}$; chlorhydrate de cystéine, $0,5 \mathrm{~g}$; tampon phosphate $0,033 \mathrm{M}$; eau distillée $1000 \mathrm{ml}$; $\mathrm{pH} \mathrm{7,0.}$

Après 3-4 jours d'incubation à $37^{\circ} \mathrm{C}$ le milieu contient des spores encore incluses dans leur sporange et des cellules végétatives. Cette culture est alors centrifugée $(20 \mathrm{mn}$ à $12000 \mathrm{~g})$ et le culot est remis en suspension dans l'eau distillée stérile après 3 lavages. Une partie est conservée à $4^{\circ} \mathrm{C}$ pour être inoculée telle quelle dans le lait. L'autre partie de la suspension est traitée pour débarrasser les spores des cellules végétatives et des sporanges selon une méthode précédemment décrite (Cerf et al., 1967). Ces spores propres sont ensuite conservées en eau distillée stérile à la température de $4^{\circ} \mathrm{C}$ et servent pour la plupart des essais comme cela est indiqué dans les résultats.

\section{3) Dénombrement des spores de $\mathrm{Cl}$. tyrobutyricum}

Les dénombrements de spores ont été effectués sur le lait avant et après bactofugation afin de déterminer le taux d'élimination dû à ce traitement. Les chauffages pratiqués au cours de la bactofugation n'étant pas suffisants pour affecter la viabilité des spores de Cl. tyrobutyricum (Cerf, Bergère et Hermier, 1967), les prélèvements ont été effectués seulement à l'entrée et à la sortie du bactofuge. On a procédé par écoulement continu d'une petite quantité de lait (environ 1 p. 1000 ) dérivée du circuit et recueillie dans un flacon stérile plongeant dans de l'eau glacée. Quelques prélèvements effectués dans le réservoir où était recueilli le lait après traitement ont montré que les résultats étaient les mêmes qu'à la sortie du bactofuge.

Les échantillons de lait (en double), préalablement chauffés à $75^{\circ} \mathrm{C}$ pendant $10 \mathrm{mn}$ pour éliminer la flore non sporulée, sont ensuite dilués puis ensemencés sur milieu liquide (R.C.M. Oxoïd à $\mathrm{pH} 6,5)$ à raison de 5 tubes par dilution. Après avoir recouvert le milieu de paraffine stérile, les tubes sont incubés à $37^{\circ} \mathrm{C}$ pendant 6 à 10 jours. On considère comme positifs les tubes présentant une croissance et un dégagement gazeux. Dans ces tubes, la présence de Cl. tyrobutyricum se traduit par une odeur butyrique caractéristique et peut être confirmée également par un examen microscopique de la culture. Le nombre de spores était alors déterminé à l'aide d'une table de MPN. 


\section{4) Influence de la centrifugation sur la répartition des spores dans le lait}

Ces essais ont été réalisés avec des laits ayant subi différents traitements qui sont précisés dans les résultats. Le lait utilisé était le même que celui qui a servi pour les essais de bactofugation ou provenait d'une étable du C.N.R.Z. Après ensemencement en $\mathrm{Cl}$. tyrobutyricum à raison de 100 à 1000 spores propres par ml et réchauffement à $40-45^{\circ} \mathrm{C}, 10 \mathrm{ml}$ de lait sont centrifugés en tubes dans une centrifugeuse de laboratoire (Sorvall RC2) dont le rotor est préalablement réchauffé à environ $40^{\circ} \mathrm{C}$. La durée et la vitesse de centrifugation sont indiqués dans les résultats. A la fin de la centrifugation la température de la centrifugeuse est abaissée à $4^{\circ} \mathrm{C}$. En outre à leur sortie les tubes sont plongés dans l'eau glacée pendant quelques minutes. On distingue alors trois couches : une couche supérieure de crème plus ou moins solidifée qui peut être prélevée à la spatule, le lait écrémé qui est siphonné et enfin un petit culot au fond du tube. Des dénombrements sont effectués sur ces trois couches par la méthode précédemment décrite (§ 3) afin de déterminer la répartition des spores.

\section{5) Mise en évidence des agglutinines}

Le pouvoir agglutinant a été déterminé sur des échantillons de lait d'origines diverses. Dans des tubes à hémolyse, $0,5 \mathrm{ml}$ de lait écrémé pur ou dilué au $1 / 2$, au $1 / 4$, au $1 / 8$, etc., dans de l'eau physiologique $(\mathrm{ClNa}, 0,9$ p. 100$)$ sont ajoutés à $0,5 \mathrm{ml}$ d'une suspension de spores ou de cellules végétatives de $\mathrm{Cl}$. tyrobutyricum ou des autres espèces testées. Cette suspension est préalablement ajustée à la densité optique de 1,0 (Beckman B à $650 \mathrm{~m} \mu$ ) par dilution dans de l'eau physiologique et contient environ $2.10^{8}$ spores par $\mathrm{ml}$. Après agitation, les tubes sont placés pendant $2 \mathrm{~h}$ au bain-marie à $37^{\circ} \mathrm{C}$ puis conservés pendant $15 \mathrm{~h}$ à la température de $4^{\circ} \mathrm{C}$. Ensuite on apprécie l'agglutination des bactéries (spores ou cellules végétatives) par un examen microscopique du contenu des tubes. Dans les résultats figurent le degré d'agglutination par lequel on désigne l'aspect de l'agglutination dans le lait non dilué $(+++$ forte, ++ moyenne, + faible) et le titre par lequel on désigne la dernière dilution du lait présentant une agglutination + . Cette technique inspirée de celle utilisée pour les bactéries lactiques (Portmann, Gâté et Auclair, 1962) permet d'observer l'agglutination aussi facilement qu'avec du sérum de lait coagulé par la présure.

Les souches de $\mathrm{Cl}$. butyricum CNRZ 528 , de $\mathrm{Cl}$. saccharobutyricum CNRZ 556, de $\mathrm{Cl}$. perfringens CNRZ 552, de $\mathrm{Cl}$. sporogenes CNRZ 543 et de Cl. tetanomorphum CNRZ 547 appartiennent à la collection du laboratoire. Les souches de $\mathrm{Cl}$. fallax et de $\mathrm{Cl}$. multifermentans proviennent de l'Institut Pasteur de Lille. Les spores de $\mathrm{Cl}$. butyricum et de $\mathrm{Cl}$. saccharobutyricum ont été préparées suivant une technique précédemment décrite (Cerf et al., 1967). Les cellules végétatives de toutes les espèces ont été obtenues par culture sur 
milieu RCM, récoltées par centrifugation et mises en suspension dans de l'eau physiologique.

\section{RESULTATS}

\section{1) Influence de la bactofugation sur l'élimination des spores de Cl. tyrobutyricum}

Pour déterminer le taux d'élimination, la bactofugation a été effectuée sur des laits contenant de 100 à 500 spores de $\mathrm{Cl}$. tyrobutyricum par $\mathrm{ml}$. Ces nombres ont été choisis de façon qu'après la bactofugation le lait contienne encore un nombre de spores suffisant pour permettre une numération précise.

Puisqu'il a été montré précédemment que la température de bactofugation pouvait avoir une influence sur le taux d'élimination des bactéries (Simonart et Debeer, 1954), nous avons effectué des essais à différentes températures comprises entre $60^{\circ} \mathrm{C}$ et $83^{\circ} \mathrm{C}$. Il faut cependant noter que ces traitements thermiques n'ont pas d'effet sur la viabilité des spores de Cl. tyrobutyricum (Cerf et al., 1967) que l'on retrouve d'ailleurs quantitativement dans les boues du bactofuge.

\section{TABLEAU 1}

Elimination des spores de Cl. tyrobutyricum par bactofugation du lait à différentes températures

\begin{tabular}{|c|c|c|c|c|c|}
\hline $\begin{array}{l}\text { Température de } \\
\text { bactofugation }\end{array}$ & $60^{\circ} \mathrm{C}$ & $65^{\circ} \mathrm{C}$ & $70^{\circ} \mathrm{C}$ & $75^{\circ} \mathrm{C}$ & $82^{\circ} \mathrm{C}$ \\
\hline Nombre d'essais & 6 & 11 & 6 & 3 & 2 \\
\hline p. 100 d'élimination $\ldots \ldots \ldots$. & $\begin{array}{l}90 \\
96,4 \\
96,4 \\
97,6 \\
98,4\end{array}$ & $\begin{array}{l}91,4 \\
92,5 \\
93,0 \\
95,8 \\
96,5 \\
97,8 \\
98,0 \\
98,4 \\
98,5 \\
99 \\
99,6\end{array}$ & $\begin{array}{l}97 \\
99,0 \\
99,0\end{array}$ & $\begin{array}{l}97,5 \\
98,0\end{array}$ & $\begin{array}{l}99,6 \\
99,8\end{array}$ \\
\hline p. 100 moyenne d'élimination & 95,8 & 96,4 & 97,5 & 97,1 & 99,7 \\
\hline
\end{tabular}

Nombre de spores propres de Cl. tyrobutyricum souche CNRZ 510 avant bactofugation, compris entre 100 et 500 par $\mathrm{ml}$ de lait. Débit du bactofuge : 6000 1/h. 
Comme le montrent les-résultats du tableau 1, les pourcentages d'élimination moyens obtenus ne varient pas beaucoup lorsque la température est comprise entre $60^{\circ} \mathrm{C}$ et $75^{\circ} \mathrm{C}$, mais ils augmentent notablement et dépassent 99 p. 100 lorsque la température est supérieure à $80^{\circ} \mathrm{C}$. Par contre d'un essai à l'autre les valeurs du pourcentage d'élimination sont assez dispersées, et cette dispersion semble d'autant plus grande que la température de bactofugation est plus faible.

Des essais à un débit plus faible, $4000 \mathrm{l}$ au lieu de $6000 \mathrm{l} / \mathrm{h}$ et à la température de $65^{\circ} \mathrm{C}$ n'ont pas permis d'obtenir un résultat meilleur, la réduction du nombre de spores est seulement de 97,6 p. 100 au lieu de 96,9 p. 100 . Le fait d'ensemencer le lait avec des spores encore incluses dans les restes des cellules-mères plutôt qu'avec des spores propres ou le fait de changer de souche de $C l$. tyrobutyricum ne modifient pas les résultats obtenus.

TABLEAU 2

Répartition des spores de $C$. tyrobutyricum souche CNRZ 510 dans le lait ayant subi une centrifugation

\begin{tabular}{|c|c|c|}
\hline \multirow{2}{*}{$\begin{array}{c}\text { p. } 100 \text { de spores après } \\
\text { centrifugation } \\
(9000 \mathrm{~g}-15 \mathrm{mn}) \\
\text { dans : }\end{array}$} & \multicolumn{2}{|c|}{ Traitement subi par le lait avant centrifugation } \\
\hline & $\begin{array}{c}\text { Pas de } \\
\text { traitement }\end{array}$ & $\begin{array}{c}\text { Chauffage à } 80^{\circ} \mathrm{C} \\
\text { pendant } 10 \mathrm{mn}\end{array}$ \\
\hline la crème & 91,6 & 0,05 \\
\hline le lait écrémé & 5,0 & 1,90 \\
\hline le sédiment $\ldots \ldots \ldots \ldots \ldots$ & 3,4 & 98,5 \\
\hline
\end{tabular}

\section{TABLEAU 3}

Influence du traitement subi par le lait sur la centrifugation des spores de $\mathrm{Cl}$. tyrobutyricum souche CNRZ 510

\begin{tabular}{|c|c|c|c|c|}
\hline \multirow[b]{2}{*}{$\begin{array}{l}\text { p. } 100 \text { de spore après } \\
\text { centrifugation } \\
(12000 \mathrm{~g}-30 \mathrm{~s})\end{array}$} & \multicolumn{4}{|c|}{ Traitement subi par le lait avant centrifugation } \\
\hline & $\begin{array}{c}\text { Pas de } \\
\text { traitement }\end{array}$ & $\begin{array}{l}\text { Chauffage } \\
75^{\circ} \mathrm{C}-15 \mathrm{~s}\end{array}$ & $\begin{array}{l}\text { Homo- } \\
\text { généisation } \\
225 \text { bars à } \\
64-65^{\circ} \mathrm{C}\end{array}$ & $\begin{array}{l}\text { Chauffage } \\
80^{\circ} \mathrm{C}-10 \mathrm{mn}\end{array}$ \\
\hline $\begin{array}{l}\text { Dans le mélange crème }+ \text { lait } \\
\text { écrémé sans sédiment } \ldots . .\end{array}$ & 95 & 27,5 & 8 & 2 \\
\hline Dans le sédiment .. & 5 & 62,5 & 92 & 98 \\
\hline
\end{tabular}




\section{TABLEAU 4}

Agglutination des spores de Cl. tyrobutyricum souche CNRZ 510 par des laits soumis à différents traitements

\begin{tabular}{|c|c|c|c|c|c|c|c|c|c|}
\hline & \multicolumn{3}{|c|}{ Laits chauffés } & \multirow{2}{*}{$\begin{array}{l}\text { Lait homogénéisé } \\
225 \text { bars }-65^{\circ} \mathrm{C}\end{array}$} & \multicolumn{5}{|c|}{ Laits bactofugés à différentes températures } \\
\hline & $\begin{array}{c}75^{\circ} \mathrm{C} \\
15 \mathrm{~s}\end{array}$ & $\begin{array}{c}80^{\circ} \mathrm{C} \\
15 \mathrm{~s}\end{array}$ & $\begin{array}{c}80^{\circ} \mathrm{C} \\
10 \mathrm{mn}\end{array}$ & & $65^{\circ} \mathrm{C}$ & $70^{\circ} \mathrm{C}$ & $75^{\circ} \mathrm{C}$ & $78^{\circ} \mathrm{C}$ & $82^{\circ} \mathrm{C}$ \\
\hline $\begin{array}{c}\text { Avant traitement : } \\
\text { Titre } . . . . . . . . . .\end{array}$ & $1 / 8$ & $1 / 8$ & $1 / 8$ & $1 / 8$ & $1 / 16$ & $1 / 16$ & $1 / 32$ & $1 / 16$ & $1 / 16$ \\
\hline $\begin{array}{l}\text { Après traitement : } \\
\text { Degré d'agglutination* }\end{array}$ & +++ & + & - & - & +++ & +++ & $\begin{array}{c}++ \\
\stackrel{+}{a} \\
+++\end{array}$ & $\begin{array}{c}+ \\
\stackrel{a}{a} \\
++\end{array}$ & + \\
\hline Titre .... & $1 / 8$ & 1 & 0 & 0 & $1 / 16$ & $\begin{array}{c}1 / 8 \\
\grave{a} \\
1 / 16\end{array}$ & $\begin{array}{l}1 / 16 \\
\grave{a} \\
1 / 32\end{array}$ & $\begin{array}{c}1 / 2 \\
\mathrm{a} \\
1 / 4\end{array}$ & 1 \\
\hline
\end{tabular}

* Avant traitement on obtenait une agglutination +++ dans tous les laits. 


\section{2) Rôle des agglutinines du lait dans la centrifugation des spores de $\mathrm{Cl}$. tyrobutyricum}

a) Influence du traitement subi par le lait sur la répartition des spores après centrifugation

Lorsqu'on laisse au repos pendant $15 \mathrm{~h}$ à $4^{\circ} \mathrm{C}$ du lait cru ensemencé en spores de $\mathrm{Cl}$. tyrobutyricum on constate que la plupart d'entre elles (70 à 90 p. 100) se retrouvent dans la couche de crème. Comme le montrent les résultats du tableau 2 , on retrouve le même phénomène lorsque le lait est soumis à une force centrifuge suffisante pour sédimenter complètement les spores d'une suspension aqueuse. Plus de 90 p. 100 des spores sont rassemblées dans la crème, le reste se retrouvant dans le lait écrémé et le culot. Par contre, si le lait est chauffé à $80^{\circ} \mathrm{C}$ pendant $10 \mathrm{mn}$ et refroidi à $45^{\circ} \mathrm{C}$ avant centrifugation, la répartition des spores est inversée et la plupart d'entre elles sont rassemblées dans le culot. Les résultats sont les mêmes lorsque les spores utilisées n'ont pas été nettoyées.

Après une centrifugation un peu plus forte mais relativement brève, et comparable à celle qui est réalisée au cours de la bactofugation, on constate que le mélange lait écrémé + crème contient encore 95 p. 100 des spores si le lait n'a pas été chauffé, 27,5 p. 100 s'il a subi une pasteurisation de $30 \mathrm{~s}$ à $75^{\circ} \mathrm{C}$, seulement moins de 10 p. 100 s'il a été préalablement homogénéisé ou surtout chauffé à $80^{\circ} \mathrm{C}$ pendant $10 \mathrm{mn}$ (tableau 3 ).

Ces résultats montrent bien que l'élimination des spores de $\mathrm{Cl}$. tyrobutyricum par centrifugation est étroitement liée au traitement préalablement subi par le lait. En particulier des traitements susceptibles de détruire les agglutinines du lait, chauffage à $80^{\circ} \mathrm{C}$ ou homogénéisation (Auclair, 1964) permettent à la plupart des spores de sédimenter dans le culot.

\section{b) Agglutination des spores de $\mathrm{Cl}$. tyrobutyricum}

Comme on peut le supposer d'après les résultats qui précèdent, le lait contient effectivement des anticorps responsables de l'agglutination des spores de $\mathrm{Cl}$. tyrobutyricum (tableau 4 ). Le titre varie suivant l'origine du lait du $1 / 4$ au $1 / 32$ mais tous les laits examinés provoquent une agglutination très nette $(+++)$ des spores, qu'il s'agisse des laits de mélange ayant servi pour la bactofugation ou des laits provenant de diverses étables (15) à des époques différentes de l'année.

Le titre varie également en fonction du traitement subi par le lait (tableau 4), en particulier le lait n'agglutine plus ces spores soit après un chauffage de $10 \mathrm{mn}$ à $80^{\circ} \mathrm{C}$ soit après une homogénéisation ( 225 bars à $64^{\circ} \mathrm{C}-65^{\circ} \mathrm{C}$ ). Par contre, jusqu'à la température de $75^{\circ} \mathrm{C}$, les chauffages subis par le lait au cours de la bactofugation n'ont pratiquement pas d'influence sur l'agglutination des spores ; on commence à observer une nette diminution du titre seulement lors- 
que la température atteint $78^{\circ} \mathrm{C}$. Au-dessus de $80^{\circ} \mathrm{C}$ le lait n'agglutine plus les spores de Cl. tyrobutyricum (tableau 4).

Pour un lait donné, le titre est le même, que les spores soient lavées ou non. Mais il est un peu plus faible pour les cellules qui sont pourtant plus fortement agglutinées par le lait non dilué (tableau 5). Les résultats sont identiques lorsqu'on utilise les autres souches de $\mathrm{Cl}$. tyrobutyricum.

TABLEAU 5

Agglutination de différentes espèces du Clostridium

\begin{tabular}{|c|c|c|c|c|}
\hline \multirow{2}{*}{ Espèce } & \multicolumn{2}{|c|}{ Degré d'agglutination } & \multicolumn{2}{|c|}{ Titre } \\
\hline & Spores & $\begin{array}{c}\text { Cellules } \\
\text { Végétatives }\end{array}$ & Spores & $\begin{array}{c}\text { Cellules } \\
\text { Végétatives }\end{array}$ \\
\hline 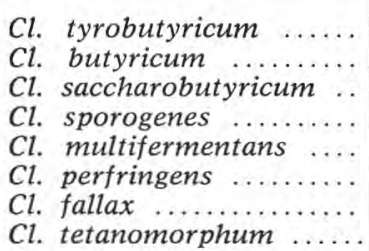 & $\begin{array}{c}+++ \\
++ \\
\pm\end{array}$ & $\begin{array}{l}+++ \\
+++ \\
++++ \\
+++ \\
+++ \\
- \\
-\end{array}$ & $\begin{array}{c}1 / 8 \\
1 / 4 \\
1\end{array}$ & $\begin{array}{c}1 / 4 \\
1 / 8 \\
1 / 32 \\
1 / 2 \\
1 / 2 \\
0 \\
0 \\
0\end{array}$ \\
\hline
\end{tabular}

* Les cellules sont généralement plus agglutinées que les spores et donnent de gros amas $(++++)$.

Cet essai a été réalisé avec un échantillon de lait de mélange.

Comme le montre le tableau 5, le lait est capable d'agglutiner d'autres espèces de Clostridium tels que $\mathrm{Cl}$. sporogenes, $\mathrm{Cl}$. multifermentans, $\mathrm{Cl}$. butyricum et $\mathrm{Cl}$. saccharobutyricum mais il n'agglutine pas $\mathrm{Cl}$. perfringens, ni $\mathrm{Cl}$. tetanomorphum. Il est d'ailleurs intéressant de remarquer, dans le cas de $\mathrm{Cl}$. saccharobutyricum, que seules les cellules végétatives sont agglutinées.

\section{DISCUSSION}

Les pourcentages d'élimination des spores de $\mathrm{Cl}$. tyrobutyricum que nous avons obtenus par bactofugation sont du même ordre de grandeur que ceux indiqués par les différents auteurs, soit pour des cultures sporulées de "butyriques " sans précision d'espèce (Lodin et al., 1965 et 1967 ; Syrjänen et al., 1963, 1964 a et b, 1965), soit pour des spores du genre Clostridium (Simonart et al., 1966). Les résultats sont d'ailleurs les mêmes que les spores soient propres ou encore incluses dans leur sporange (comme ce doit être le cas dans la pratique) et en mélange avec des cellules végétatives (culture sporulée). Toutefois il est important de remarquer que les pourcentages d'élimination et en particulier leur reproductibilité est fonction 
de la température de la bactofugation. Il sera donc nécessaire d'en tenir compte lors de l'application de ce traitement à des laits de fromagerie contaminés en spores de $\mathrm{Cl}$. tyrobutyricum (Bergère et al., 1969).

En cherchant à déterminer le rôle de la température dans la centrifugation on a constaté que les spores de $\mathrm{Cl}$. tyrobutyricum se rassemblent dans la crème et non dans le sédiment si le lait est simplement réchauffé à $45^{\circ} \mathrm{C}$ avant centrifugation. Ce phénomène qui était déjà connu pour les spores de " butyriques « (Hostettler et al., 1941 ; Sjöstrom et al., 1963), est certainement provoqué par les agglutinines du lait que nous avons mises en évidence. D'ailleurs lorsque le lait a subi un traitement capable de détruire les agglutinines (Auclair, 1964) (chauffage à $80^{\circ} \mathrm{C}$ ou homogénéisation) la centrifugation permet de recueillir les spores dans le sédiment et non plus dans la crème comme cela a déjà été constaté par Labots et Hup (1964) pour les spores de B. cereus. Comme nous l'avons vu, le lait est capable d'agglutiner non seulement les spores et les cellules végétatives de $\mathrm{Cl}$. tyrobutyricum mais aussi celles d'autres espèces de Clostridium appartenant ou non au groupe butyrique. Il contient donc au moins des anticorps agglutinants spécifiques, des antigènes communs à ces différentes espèces et provenant d'une immunisation naturelle des animaux. On sait d'ailleurs que $\mathrm{Cl}$. tyrobutyricum possède des antigènes communs à $\mathrm{Cl}$. butyricum, $\mathrm{Cl}$. sporogenes et surtout à $\mathrm{Cl}$. botulinum mais pas à $\mathrm{Cl}$. perfringens, ni à Cl. tetanomorphum (Sharpe et Goudkov, 1966).

En fait dans les laits naturellement contaminés ou faiblement ensemencés avec $\mathrm{Cl}$. tyrobutyricum, la concentration en spores est insuffisante pour qu'il y ait agglutination. Cependant par l'intermédiaire des agglutinines qui sont adsorbées sur les globules gras les quelques spores présentes dans le lait peuvent être fixées sur ces globules et, comme nous l'avons constaté, se trouver entraînées avec eux sous l'effet de la force centrifuge. Les forts pourcentages de spores éliminées dans les boues par la bactofugation peuvent donc paraître surprenants lorsque les chauffages du lait (températures inférieures à $78^{\circ} \mathrm{C}$ ) sont insuffisants pour détruire les agglutinines. Cela peut cependant s'expliquer par le fait que le bactofuge est certainement plus efficace qu'une centrifugeuse de laboratoire non seulement en raison de son mode de construction mais surtout en raison de son mode de fonctionnement. En effet dans cet appareil le lait est centrifugé à chaud. Or on sait que la quantité d'agglutinines adsorbées sur les globules gras diminue lorsqu'on élève la température du lait (Sharp et Krukovsky, 1939 ; Dunkley et Sommer, 1944). En conséquence la proportion de spores susceptibles d'être entraînées avec les globules gras (phase légère) du lait doit également diminuer lorsque la température augmente. On peut donc supposer que c'est la principale raison pour laquelle le pourcentage de spores éliminées dans le sédiment (ou boues) du bactofuge augmente avec la température et dépasse nettement celui qu'on obtient par simple centri- 
fugation sans chauffage. De plus, d'un essai à l'autre, la quantité d'agglutinines restant fixées sur les globules gras, donc susceptibles d'entraîner encore des spores, doit varier, ne serait-ce qu'en fonction de leur teneur initiale dans le lait, ce qui est certainement à l'origine de la dispersion des résultats obtenus par bactofugation.

Cette hypothèse sur le rôle des agglutinines se trouve d'ailleurs confirmée par les résultats obtenus aux températures voisines de (ou supérieures à) $80^{\circ} \mathrm{C}$. La très nette augmentation des pourcentages d'élimination obtenus par la bactofugation effectuée à ces températures ne peut en effet s'expliquer que par la destruction des agglutinines, et non plus par des variations de viscosité du lait comme ce peut être le cas à des températures plus basses (inférieures à $45^{\circ} \mathrm{C}-50^{\circ} \mathrm{C}$ ) (Simonart et Debeer, 1954). On a d'ailleurs constaté que la bactofugation à $45^{\circ} \mathrm{C}$ d'un lait préalablement chauffé à $80^{\circ} \mathrm{C}$ pendant 15 à $30 \mathrm{~s}$ donne d'aussi bons résultats que la bactofugation directement pratiquée à $80^{\circ} \mathrm{C}$. Ceci montre bien qu'à partir du moment où les agglutinines ont été détruites, la température de bactofugation a beaucoup moins d'importance sur l'élimination des spores.

Ainsi très certainement la présence d'agglutinines dans le lait constitue le principal facteur limitant l'efficacité de la bactofugation dans l'élimination des spores de $\mathrm{Cl}$. tyrobutyricum qui, malgré la viscosité du lait, devrait être plus élevée en raison du fait que leur densité diffère nettement de celle du lait, environ 1,2 pour les spores contre 1,03 pour le lait.

\section{Remerciements}

Nous remercions MM. Hermier et Mocquot pour leurs suggestions et critiques au cours de la rédaction du manuscrit, ainsi que le Dr Beerens pour les souches de Clostridium, qu'il nous a aimablement procurées.

Nous remercions également la Société Alfa-Laval qui a bien voulu installer à la Laiterie Expérimentale de Jouy-en-Josas, le bactofuge ayant servi pour ces essais.

\section{Ré su m é}

L'efficacité de la bactofugation dans l'élimination des spores de $\mathrm{Cl}$. tyrobutyricum du lait dépend de la température. De $60^{\circ} \mathrm{C}$ à $75^{\circ} \mathrm{C}$, les taux d'élimination moyens n'augmentent que de 95,8 à 97 p. 100 , mais ils dépassent 99 p. 100 lorsque la température atteint $80^{\circ} \mathrm{C}$. Par contre à une température donnée, les valeurs de ces pourcentages varient d'un essai à l'autre et cette variation est d'autant plus grande que la température de bactofugation est plus faible. 
Par ailleurs on a constaté que par centrifugation du lait à la température de $35^{\circ} \mathrm{C}$ à $40^{\circ} \mathrm{C}$ dans un appareil de laboratoire, les spores de $\mathrm{Cl}$. tyrobutyricum se rassemblent soit dans la couche de crème si le lait est cru, soit dans le sédiment si le lait a été chauffé à $80^{\circ} \mathrm{C}$ pendant $10 \mathrm{mn}$. Ce phénomène est en relation avec les anticorps qui ont été trouvés dans le lait et qui agglutinent les spores de Clostridium. Le rôle joué par ces agglutinines au cours de la bactofugation est ensuite discuté.

\section{$\mathrm{S}$ u $\mathbf{m} \mathbf{m}$ a r y \\ Bactofugation of milk and removal of Clostridium tyrobutyricum spores}

The effectiveness of bactofugation in removing $C l$. tyrobutyricum spores from milk depended on temperature. From 60 to $75^{\circ} \mathrm{C}$, the average rates of spores removed from milk only increased from 95,8 to $97 \%$, but at $80^{\circ} \mathrm{C}$ or more, they were higher than $99 \%$. However, at a given temperature, these rates were different from trial to another and the lower the temperature of bactofugation the higher this variation.

Furthermore, during centrifugation of milk at $35-40^{\circ} \mathrm{C}$ in a laboratory apparatus, most of $\mathrm{Cl}$. tyrobutyricum spores were accumulated eitner in the cream layer of raw milk or in the sediment of milk heated at $80^{\circ} \mathrm{C}$ for $10 \mathrm{~min}$. This was related to the anti-bodies which had been found in milk and which agglutinated spores of Clostridium. The effect of agglutinins to be expected during bactofugation was discussed.

Reçu pour publication en juin 1969.

\section{Bibliographie}

[1] Auclair (J.). - Les substances antibactériennes du lait cru et leur rôle en technologie laitière. Microbial Inhibitors in Food, Almqvist \& Wiksell, Stockholm, 1964.

[2] Bergère (J. L.), Gouet (Ph.), Hermier (J.) et Mocouot (G.). - Les Clostridium du groupe butyrique dans les produits laitiers. Ann. Inst. Pasteur, Lille, 1968, 19, p. 41.

[3] Bergère (J. L.), Rousseaux (P.), Ducruet (P.), Mocouot (G.), Hermier (J.), Gouet (Ph.) et Zelter (S. Z.). - Expérimentation sur la fabrication d'Emmental et de Comté avec du lait de vaches nourries à l'ensilage A.I.V. I. Aspects technologiques. Bull. Tech. Inf., 1969, 239, p. 357.

[4] Bergère (J. L.), Le Bars (D.) et VASSAL (L.). - Influence de la bactofugation sur l'apparition du gonflement butyrique dans le fromage d'Emmental, Revue Laitière Française, 1969, 267, p. 463.

[5] Cerf (O.), Bergère (J. L.) et Hermier (J.). - Thermorésistance des spores de Clostridium tyrobutyricum et Clostridium butyricum. J. Dairy Res., 1967, 34 , p. 221.

[6] Dunkley (W. L.) and Sommer (H. H.). - Res. Bull. Wisc. Agric. exp. Sta., $1944,151$. 
[7] Hostettler (H.), Sahli (K) und Binz (M.). - Beitrag zur Methodik des Nachweises anaerober Buttersaürebazillen in Milch. Mitt. Geb. Lebensmittelunters. U. Hyg., 1941, 32, p. 76.

[8] Labots (H.) and Hup (G.). - Bacillus cereus in raw and pasteurised milk. I. Enumeration of B. cereus in milk. Neth. Milk Dairy J., 1964, 18, p. 122.

[9] Lodin (J. O.). - Baktofugering av ystmojölk. Svenska Mejeritidn., 1965, 57, p. 335.

[10] Lodin (L. O.), Lindgren Britta och Nilsson (R.). - Baktofugering av ystmjölk. Svenska Mejeritidn., 1967, 59, p. 253.

[11] Peltola (E.), Syrjanen (H.). - Bakteeriseparointi. IV. Emmentaljuuston valmistus. Karjantuote, 1965, 48, (13-14), p. 363.

[12] Portmann (A.), Gaté (Y.) et Auclair (J.). - Influence de la lactoperoxydase et des agglutinines du lait sur l'activité des bactéries des levains thermophiles. $X V I^{\mathrm{e}}$ Cong. Intern. Laiterie, 1962, B, p. 729.

[13] Sharp (P. F.) and Krukovsky (V. N.). - Differences in adsorption of solid and liquid fat globules as influencing the surface tension and creaming of milk. J. Dairy Sci., 1939, 22, p. 743.

[14] Sharpe (M. E.) and Goudkov (A. V.). - Serological characteristics of some Clostridia isolated from milk and cheese, XVII ${ }^{\circ}$ Cong. Intern. Laiterie, $1966, D$, p. 625.

[15] Simonart (P.) et Debeer (G.). - Recherches en vue d'améliorer la qualité microbiologique des laits par ultracentrifugation. Neth. Mitk Dairy J., 1953, 7 , p. 117.

[16] Simonart (P.) et Debeer (G.). - Centrifugation et teneur en bactéries du lait. Neth. Milk Dairy J., 1954, 8, p. 101.

[17] Simonart (P.), Poffé (R.), Weckx (M.). - Double supercentrifugation bactérienne du lait. Le Lait, 1959, 39, p. 129.

[18] Simonart (P.), Poffé (R.), Weckx (M.) et Van Reusel (A.). - Bactofugation et flore bactérienne du fromage "Gouda ». XVII Cong. Intern. Laiterie, $1966, D$, p. 21.

[19] SJöstRöM (G.) et OLSson (H.). - Undersökningar över en centrifugeringsmetod för bestänning av laktatjäsande smörsyrabakteriers sporer i leverantörsmjölk. Svenska Mejeritidn., 1963, 55, p. 487-508.

[20] SyrJanen (H.). - Bakteeriseparointi. I. Vaikutus juustolamaidon bakteereihin ja ominaisuuksiin. Karjantuote, 1963, 9, p. 216.

[21] Syruanen (H.). - Bakteeriseparointi. II. Edamjuuston valmistus. Karjantuote, 1964,1, p. 1.

[22] Syrjanen (H.), - Bakteeriseparointi. III. Emmentaljuuston valmistus. Karjantuote, 1964, 5-6, p. 113.

[23] Thomé (K. E.) and Swartling (P.). - Influence of silage quality on cheesemilk and cheese quality. XIII ${ }^{\mathrm{e}}$ Cong. Intern. Laiterie, 1953, 11, p. 69. 